\title{
„Jeder an Finanzinvestoren ausgeschüttete Euro fehlt dem Gesundheitswesen als Ganzes und damit der Solidargemeinschaft"
}

Finanzinvestoren werden auf den globalen Gesundheitsmärkten und auch im deutschen Gesundheitssystem immer aktiver. Was sind die Folgen dieser Entwicklung für das hiesige Gesundheitswesen und besonders die Radiologie? Über den Einfluss privater Investoren auf die Radiologie haben wir mit Professor Hermann Helmberger gesprochen, Leiter des Zentrums für Radiologie und Nuklearmedizin am Klinikum Dritter Orden München-Nymphenberg, Mitglied der Steuerungsgruppe des CAFRAD der Deutschen Röntgengesellschaft (DRG) und des Bundesvorstandes des Berufsverbandes Deutscher Radiologen (BDR).

Professor Helmberger, kapitalstarke private Finanzinvestoren sind etwa im Pflegebereich und in der Krankenhauslandschaft schon lange tätig und seit einiger Zeit auch im Bereich ambulanter Praxen. Wann hat diese Entwicklung angefangen und von wem wird sie vorangetrieben?

Die Entwicklung, dass finanzstarke Investorengruppen in die Medizin drängen, beobachten wir in der Dialysebehandlung und der Labormedizin bereits seit mehr als 20 Jahren. Als erste Facharztgruppen wurden dann im Verlauf die Augenheilkunde und die Zahnmedizin erfasst. Seit etwa 4 Jahren ist diese Entwicklung schließlich auch in der Radiologie festzustellen. Hinter dieser Entwicklung stehen zum einen Investitionsfirmen, die sich in der Medizin Möglichkeiten zur Geldanlage mit höheren Renditen erhoffen als derzeit auf dem Geldmarkt realisierbar ist. Zum anderen interessieren sich medizinische Großkonzerne für die Radiologie, um durch ein möglichst komplettes Angebotsspektrum Marktanteile in toto zu gewinnen.

Was sind die Gründe für diese Entwicklung und welche ambulanten medizinischen Konstrukte oder Organisationsformen folgen daraus?

Als Gründe sind eindeutig die derzeitige globale Niedrigzinspolitik mit geringen Renditeaussichten anzusehen und der Wunsch, vorhandenes Kapital in großem Umfang alternativ anzulegen. All diese Leistungsanbieter sind zu $100 \%$ in Form Medizinischer Versorgungszentren (MVZ) organisiert.

Auch die Radiologie gehört zu den Facharztsparten, in denen Finanzinvestoren besonders aktiv sind. Warum ist das so?
Bekanntermaßen werden in der Radiologie im Vergleich zu anderen Fachgebieten hohe Umsätze realisiert, was mit der vergleichsweise hohen Kostenquote zusammenhängt. Die Erwartungen der Investoren beruhen auf der Annahme, dass durch eine Verbesserung der Kosteneffizienz in der Radiologie hohe Gewinnmargen erreicht werden können. Dabei wird allerdings häufig übersehen, dass die radiologischen Praxen in Deutschland bereits heute über sehr effiziente Organisationsstrukturen zur Kostenreduktion verfügen und damit an der Belastungsgrenze arbeiten. Ein weiterer Aspekt ist die Altersstruktur der inhabergeführten Praxen; auch sie wirkt sich günstig für externe Investoren aus. Praxisabgaben von Arzt zu Arzt gestalten sich aus vielfältigen Gründen schwierig. Ein unkomplizierter Verkauf an einen Investor hat demgegenüber vordergründig Vorteile.

Könnten Sie uns die aktuelle Situation für ambulant tätige, niedergelassene Radiologinnen und Radiologen beschreiben?

Wie in vielen Teilen der Medizin sind die politischen Rahmenbedingungen auch in der Radiologie zunehmend unsicher und die Zukunft damit immer weniger planbar. An- 
gesichts des angesprochenen hohen Investitionsbedarfs in unserem Fach steht jeder Praxisinhaber in einer hohen persönlichen Verantwortung mit immer schlechter kalkulierbarem Marktwert der Praxis in der Zukunft. Nicht zuletzt diese Aussichten veranlassen immer mehr Kolleginnen und Kollegen zunächst oder auch langfristig, ein Anstellungsverhältnis in einer Praxis einzugehen und nicht Anteilseigner zu werden.

Wie schätzen Sie die Folgen dieser Entwicklung für die Radiologie beziehungsweise für die radiologische Versorgungsqualität für Patientinnen und Patienten ein?

Finanzinvestoren interessieren sich primär weniger für die radiologische Leistung als vielmehr für die zu erzielende Rendite. Dies führt durch Selektion zum Verschwinden geringer vergüteter Leistungen aus dem diagnostischen Spektrum und damit einer erheblichen Verschlechterung der allgemeinen Versorgungsqualität. Warnende Beispiele hierzu gibt es etwa in der bereits angesprochenen Zahnheilkunde und sind im stationären Bereich generell seit Längerem zu beobachten. Für die großen Medizinkonzerne steht das Gewinnen von Marktanteilen und weniger die radiologische Leistung im Vordergrund. Folgen hiervon sind eine marktbeherrschende Stellung einzelner sogenannter
Portalpraxen mit allen Nachteilen monopolistischer Strukturen. Gefördert wird dies durch die Möglichkeit der Großkonzerne aufgrund der dann verfügbaren Datenfülle auch im Bereich der KI-gestützten Assistenzsysteme entscheidenden Einfluss zu gewinnen.

Welche Handlungsspielräume sehen Sie noch beziehungsweise was kann die Radiologie dieser Entwicklung entgegensetzen?

Im Vordergrund muss eine patientenorientierte Versorgung stehen. Die hierzu erforderliche hohe diagnostische Qualität kann in der ambulanten Radiologie nur durch die inhabergeführte Praxis oder das ärztlich geleitete MVZ auf Dauer gewährleistet werden. Leider steht die Politik der aktuellen Entwicklung hin zu rein finanziell getriebenen Strukturen nicht ablehnend gegenüber. Übersehen wird dabei, dass jeder im Rahmen der Renditemaximierung an Finanzinvestoren ausgeschüttete Euro dem Gesundheitswesen als Ganzes und damit der Solidargemeinschaft fehlt.

Um abschließend einen Teil des Titels der Veranstaltung vom 25. September 2021 auf dem Deutschen Röntgenkongress aufzugreifen: Haben Ihrer Ansicht nach die bisherigen radiologischen Organisationsformen - insbesondere im ambulanten
Bereich - noch eine Zukunft im Zeitalter der Finanzinvestoren?

Auch die Finanzinvestoren nutzen die Organisationsform des MVZ. Dennoch ist der häufig gehörte Satz „Ohne Investoren gibt es keine Zukunft in der Radiologie“ so sicher nicht richtig. Es wird vermehrter Anstrengungen zur Kooperation auch sektorübergreifend zwischen ambulantem und stationärem Bereich bedürfen. Der BDR als Berufsverband aller Radiologen in Niederlassung und Klinik und CAFRAD als Vertretung der radiologischen Chefärzte bieten gute Plattformen, diese Kooperationen zu fördern und inhabergeführte Leistungsanbieter zu erhalten.

Am 25. September 2021 befasste sich die Veranstaltung „Quo vadis Radiologie 2021 - Haben unsere bisherigen Organisationsformen eine Zukunft im Zeitalter der Finanzinvestoren - Eine Diskussionsrunde aus dem BDR und CAFRAD“ auf dem Deutschen Röntgenkongress mit dem Thema Finanzinvestoren und Folgen für die Radiologie. Informationen dazu erhalten Sie über www.drg.de. 